\title{
Erratum to: Effects of Distance and Shape on the Estimation of the Piecewise Growth Mixture Model
}

\author{
Yuan $\mathrm{Liu}^{1,2} \cdot$ Hongyun $\mathrm{Liu}^{3}$ \\ Published online: 31 May 2019 \\ (C) The Classification Society 2019
}

\section{Erratum to: Journal of Classification https://doi.org/10.1007/s00357-018-9291-9}

The authors missed an important reference "Liu, Luo, \& Liu, 2014" on the original version of this article. With this, this should be added on text to read:

"We referred to the PGMM model based on the reading development indicators of ECLS-K as guides setting the above residual values (e.g., see Liu et al. 2014; Liu et al. 2016; Kainz and Vernon-Feagans 2007; Tourangeau et al. 2009)."

In reference list, "Liu, Y., Luo, F.,\& Liu, H. (2014). Factors of piecewise growth mixture model: distance and pattern. Acta Psychologica Sinica. 46(9). 1400-1412. doi:10.3724/ SP.J.1041.2014.01400" should be listed.

This research has also been supported by the Fundamental Research Funds for the Central Universities (Project No. SWU1809677).

Publisher's Note Springer Nature remains neutral with regard to jurisdictional claims in published maps and institutional affiliations.

The online version of the original article can be found at https://doi.org/10.1007/s00357-018-9291-9

Hongyun Liu

hyliu@bnu.edu.cn

Yuan Liu

lyuuan@swu.edu.cn

1 Faculty of Psychology, Southwest University, No. 2 Tiansheng Rd., Beibei District, Chongqing, China

2 Key Laboratory of Cognition and Personality, Ministry of Education, Chongqing, China

3 School of Psychology, Beijing Normal University, No. 19 Xinjiekouwai St., Hai Dian District, Beijing, China 